\title{
Physical demands analysis of soccer players during the extra-time periods of the UEFA Euro 2016
}

\author{
A Kubayi, DPhil; A Toriola, $\mathrm{PhD}$ \\ Department of Sport, Rehabilitation and Dental Sciences, Faculty of Science, \\ Tshwane University of Technology, Pretoria, South Africa
}

\section{Corresponding author: A Kubayi (kubayina@tut.ac.za)}

Background: Despite the importance of extra-time (ET) in determining success in the knockout stages of tournaments, there is scant information on the physical demands of ET on soccer players.

Methods: This study investigated the physical demands of all soccer players $(n=59)$ who completed four matches that went to ET at the 2016 UEFA Euro Championship. Players were categorised as follows: central defenders (CDs), wide defenders (WDs), central midfielders (CMs), wide midfielders (WMs) and attackers (ATs). Match activities were captured using a validated camera tracking system (InStat ${ }^{\circledR}$ ). Descriptive statistics and repeated measures one-way analysis of variance (ANOVA) were used to analyse the data.

Results: The findings showed that total distances covered by players during matches decreased by $13 \%$ from the first half of the game $(113 \pm 10 \mathrm{~m} / \mathrm{min})$ to ET $(98 \pm 10 \mathrm{~m} / \mathrm{min})$. Concerning playing positions, a decline in total distances covered during matches was more apparent among midfielders than players in other field positions. A repeated measures ANOVA, with a Greenhouse-Geisser correction, showed that the mean total distances differed significantly between halves of the game $[F(1.54,83.28)=121.97, p<0.001]$.

Conclusion: Intervention strategies needed to sustain soccer players' physical performance during ET periods and of postmatch recovery modalities warrant further investigation.

Keywords: distance, physical performance, substitution, tournament

\section{S Afr J Sports Med 2018;30:1-3. DOI: 10.17159/2078-516X/2018/v30i1a4842}

A soccer match is played by two teams, each consisting of 10 outfield players and a goalkeeper. Coaches are only allowed to make three substitutions during a game - for injured players or for tactical reasons. The regulation time for a soccer match is 90 minutes, consisting of two halves of 45 minutes each, with a 15-minute half-time break. Typically, one to three minutes of referee's optional time or longer are added to each half to allow for recovery of time lost either to injury or for prolonged disruption of the game during normal regulation time. During the knockout stages of soccer matches, the game is extended by 30 minutes of extra-time (ET) if neither side has secured a decisive victory within the normal 90-minute regulation period. ${ }^{[1-4]}$

Between 1986 and 2014, 35\% of FIFA World Cup matches in the knockout stages required ET. ${ }^{2]}$ The need for ET in soccer tournaments is becoming more common, for example, $50 \%$ of knockout matches at the 2014 FIFA World Cup required 120 minutes of match play compared to $25 \%$ at the 2002 and 2010
FIFA World Cup tournaments respectively, as well as 38\% at the 2006 FIFA World Cup. ${ }^{[2]}$ However, the physical demands of ET on elite soccer players have rarely been studied. This is somewhat surprising considering the role of this additional period of play in determining success in soccer tournaments. [3]

To date, two studies were conducted by Lago-Peñas et al. ${ }^{[4]}$ and Russell et al. ${ }^{[5]}$ who focused on the impact of the ET period on physical performance in professional soccer. These studies concluded that the total distance covered high-intensity running, maximal running speed and the number of sprints were reduced in ET compared to the first and second halves of the matches. Despite the novelty of the information provided by these studies, ${ }^{[4,5]}$ there is scant evidence for the physical demands of soccer players during the ET period, especially in a popular tournament such as the UEFA Euro.

Therefore, research is clearly needed in this regard because the UEFA Euro is one of the most important tournaments in world soccer; for example, four out of the last five FIFA World Cup competitions were won by European teams. [6] Furthermore, a better understanding of the movement demands of the ET period could provide information on the tactical preparations and recovery practices of soccer players when matches that require this additional period of competition are either anticipated or have occurred. [5] Therefore, the purpose of this study was to analyse the influence of the ET period on the physical demands of soccer players during the 2016 UEFA Euro Championship.

\section{Methods}

\section{Match data and participants}

The sample consisted of four matches from six teams that went to ET during the 2016 UEFA Euro Championship. All players (with the exception of the goalkeepers) who finished the entire 120 minutes of the game were included in the analyses $(n=59)$. Players were categorised as follows: central defenders (CDs: $\mathrm{n}=16$ ), wide defenders (WDs: $\mathrm{n}=12$ ), central midfielders (CMs: $\mathrm{n}=10$ ), wide midfielders (WMs: $\mathrm{n}=9$ ) and attackers (ATs: $\mathrm{n}=12$ ). The study received ethical clearance from the Ethics Committee of Tshwane University of Technology, South Africa.

\section{Data collection}

Match physical indicators were recorded using the camera tracking system, InStat Scout Video Analysis, Russia. Match data were captured with video cameras, installed in pairs on each of the two main stands of the stadium. The accuracy of the InStat tracking system has been reported in a previous study. ${ }^{7]}$ In addition, the physical indicator variables were categorised as follows: walking $(0-7 \mathrm{~km} / \mathrm{h})$, jogging $(7.1-14.5 \mathrm{~km} / \mathrm{h})$, running $(14.6-20 \mathrm{~km} / \mathrm{h})$, high-speed running $(20.1-25 \mathrm{~km} / \mathrm{h})$, and sprinting $(>25 \mathrm{~km} / \mathrm{h})$. Total distance represented the summation of distances in all categories. Absolute distances (m) were converted to the relative distance covered per unit of time $(\mathrm{m} / \mathrm{min}) \cdot{ }^{[4]}$ 


\section{Statistical analysis}

Data were expressed as means and standard deviations $(\mathrm{M} \pm \mathrm{SD})$. Repeated measures one-way analysis of variance (ANOVA) were conducted on the distances covered in the matches played based on the various playing positions $(\mathrm{CD}$, WD, CM, WM, and AT) during the first and second halves, as well as for the ET period. Bonferroni post-hoc analyses were further carried out to identify which of the distances covered were substantially different, and the significance level was set at 0.05 . All statistical analyses were performed using IBM SPSS software (version 25.0).

\section{Results}

Table 1 presents the physical demands of players during the first and second halves of the matches as well as the ET period. Overall, total distances covered by players during the matches decreased by $13 \%$ from the first half of the game $(113 \pm 10 \mathrm{~m} / \mathrm{min})$ to ET $(98 \pm 10 \mathrm{~m} / \mathrm{min})$. With regard to playing positions, a reduction of total distances covered during the matches was more apparent among the midfielders (WDs) than players in other field positions. For example, the total distance covered by the WMs was reduced by $17 \%$ in the first half of the game $(119 \pm 10 \mathrm{~m} / \mathrm{min})$ to ET $(99 \pm 9 \mathrm{~m} / \mathrm{min})$ (Table 1$)$. A repeated measures ANOVA, with a Greenhouse-Geisser correction, showed that the mean total distances differed significantly between the halves of the game $[F(1.54,83.28)=$ 121.97, $p<0.00]$.

The reduction of the sprinting distance was greater among ATs than other players (i.e. CDs, CMs, WMs, and CMs). Specifically, the sprinting distance decreased from the first half of the game $(3 \pm 1 \mathrm{~m} / \mathrm{min})$ to the ET period $(1 \pm 1 \mathrm{~m} / \mathrm{min})$. A repeated measures ANOVA, with a Greenhouse-Geisser correction, indicated that the sprinting distance was significant between the halves of the game $[F(1.74,93.96)=$
4.25, $p<0.02$ ]. A Bonferroni post-hoc test showed that the average sprinting distance for the ATs was significantly different from those of the CDs, CMs, and WDs.

\section{Discussion}

This study investigated the physical demands of soccer players during the ET periods of the 2016 UEFA Euro Championship. The results showed that total distances covered by the players during the matches decreased by $13 \%$ from the first half to the ET period. This is a faster rate of decline than that reported by Russell et al. ${ }^{[5]}$ who stated that the mean distance covered by players reduced by $\sim 10 \%$ in the ET period compared to the first and second halves. It has been speculated that players who fail to perform the expected work rates in a game may be experiencing a decline in aerobic capability due to fatigue. ${ }^{[8]}$

Furthermore, the decrease in the total distance covered during the matches affected all playing positions, a finding which supports that of Lago-Peñas et al. ${ }^{[4]}$ This reduction was more apparent among midfielders (WDs) than defenders. This could be attributed to the longer distances covered by the midfielders in the first and second halves of the matches in the present study, which might have reduced the distances covered during the ET period. A greater reduction in high-intensity running (i.e. sprinting) was more evident among the ATs than players in other positions. As Bradley et al. ${ }^{[9]}$ argued, the ATs are required to maintain a high level of activity, even when not directly involved in play, in order to create space to receive passes or to pressurise opponents into making unforced errors.

Although not ascertained in the current study, previous research has demonstrated that technical skills decrease considerably from the first half of a match to the ET period. For example, Harper et al. [3] reported that passing performance was reduced by more than $20 \%$ during the ET when compared to the first half of the game. These findings may suggest that

Table 1. Physical demands of players during first and second halves of the matches as well as extra-time periods

\begin{tabular}{|c|c|c|c|c|c|c|c|c|c|}
\hline & \multicolumn{3}{|c|}{ All $(n=59)$} & \multicolumn{3}{|c|}{$\mathrm{CD}(\mathrm{n}=16)$} & \multicolumn{3}{|c|}{ WD (n= 12) } \\
\hline & 1st half & 2nd half & ET & 1st half & 2nd half & ET & 1st half & 2nd half & ET \\
\hline Total distance (m/min) & $113 \pm 10$ & $107 \pm 9$ & $98 \pm 10$ & $104 \pm 6$ & $98 \pm 5$ & $91 \pm 7$ & $111 \pm 3$ & $106 \pm 5$ & $99 \pm 10$ \\
\hline Walking (m/min) & $37 \pm 4$ & $37 \pm 4$ & $38 \pm 4$ & $38 \pm 4$ & $39 \pm 4$ & $39 \pm 6$ & $38 \pm 4$ & $37 \pm 3$ & $38 \pm 2$ \\
\hline Jogging (m/min) & $46 \pm 7$ & $43 \pm 6$ & $37 \pm 7$ & $43 \pm 3$ & $40 \pm 5$ & $34 \pm 5$ & $43 \pm 4$ & $42 \pm 5$ & $38 \pm 6$ \\
\hline Running (m/min) & $19 \pm 5$ & $17 \pm 5$ & $15 \pm 4$ & $15 \pm 4$ & $13 \pm 4$ & $12 \pm 4$ & $18 \pm 3$ & $16 \pm 4$ & $14 \pm 5$ \\
\hline High-speed running (m/min) & $9 \pm 3$ & $8 \pm 3$ & $7 \pm 2$ & $7 \pm 2$ & $6 \pm 2$ & $5 \pm 2$ & $10 \pm 2$ & $9 \pm 3$ & $7 \pm 2$ \\
\hline \multirow[t]{3}{*}{ Sprinting $(\mathrm{m} / \mathrm{min})$} & $2 \pm 3$ & $2 \pm 1$ & $1 \pm 1$ & $1 \pm 1$ & $1 \pm 1$ & $1 \pm 1$ & $2 \pm 1$ & $2 . \pm 1$ & $2 \pm 1$ \\
\hline & \multicolumn{3}{|c|}{$\mathrm{CM}(\mathrm{n}=10)$} & \multicolumn{3}{|c|}{$W M(n=9)$} & \multicolumn{3}{|c|}{$\operatorname{AT}(n=12)$} \\
\hline & 1st half & 2nd half & ET & 1st half & 2nd half & ET & 1st half & 2nd half & ET \\
\hline Total distance (m/min) & $118 \pm 12$ & $113 \pm 9$ & $104 \pm 10$ & $119 \pm 10$ & $110 \pm 8$ & $99 \pm 9$ & $115 \pm 7$ & $109 \pm 7$ & $103 \pm 6$ \\
\hline Walking (m/min) & $34 \pm 2$ & $34 \pm 4$ & $36 \pm 3$ & $36 \pm 5$ & $36 \pm 5$ & $36 \pm 4$ & $37 \pm 3$ & $38 \pm 3$ & $39 \pm 4$ \\
\hline Jogging (m/min) & $53 \pm 7$ & $48 \pm 6$ & $42 \pm 7$ & $49 \pm 8$ & $44 \pm 7$ & $39 \pm 7$ & $46 \pm 7$ & $43 \pm 7$ & $39 \pm 7$ \\
\hline Running (m/min) & $23 \pm 7$ & $21 \pm 5$ & $17 \pm 4$ & $23 \pm 5$ & $19 \pm 4$ & $16 \pm 4$ & $19 \pm 3$ & $17 \pm 2$ & $16 \pm 3$ \\
\hline High-speed running ( $\mathrm{m} / \mathrm{min})$ & $11 \pm 4$ & $9 \pm 2$ & $8 \pm 2$ & $10 \pm 3$ & $10 \pm 4$ & $7 \pm 3$ & $10 \pm 1$ & $9 \pm 1$ & $9 \pm 2$ \\
\hline Sprinting (m/min) & $1 \pm 1$ & $1 \pm 1$ & $1 \pm 1$ & $1 \pm 1$ & $1 \pm 1$ & $1 \pm 1$ & $3 \pm 1$ & $2 \pm 0$ & $1 \pm 1$ \\
\hline
\end{tabular}

Data expressed as mean $\pm S D$

Physical indicator variables were categorised as follows: walking $(0-7 \mathrm{~km} / \mathrm{h})$, jogging $(7.1-14.5 \mathrm{~km} / \mathrm{h}), \mathrm{running}(14.6-20 \mathrm{~km} / \mathrm{h})$, high-speed running (20.1-25 $\mathrm{km} / \mathrm{h})$, and sprinting $(>25 \mathrm{~km} / \mathrm{h})$. Total distance represented the summation of distances in all categories.

CD, Central defender; WD, Wide defender; CM, Central midfielder; WM, Wide midfielder; AT, Attacker; ET, Extra-time 
match-related fatigue had a greater influence on the players technical ability to get involved with the ball than on their skill proficiency. ${ }^{[10]}$ Thus, soccer coaches and scientists could use the results of this study to inform the current practices in a deeper understanding of the influence of the ET on the physical demands of the game and develop intervention strategies to delay the onset of fatigue among players. [2] However, few studies have investigated the influence of matches requiring the ET on players' recovery modalities and subsequent performances in games during congested fixture schedules. [2]

\section{Conclusion}

This study found that the physical demands of soccer players, irrespective of their playing position, decreased from the first half to the ET period of the matches played. In addition, a reduction of total distances covered during the matches was more apparent among the midfielders than players in other field positions. Based on the findings of the current study, soccer governing bodies (e.g. FIFA) should consider introducing a fourth substitution for matches that extend to the ET. This view is consistent with that of Harper et al. [2] which suggested that such a substitution could minimise the effects of fatigue, reduce the risk of load-related injuries and improve player performance.

\section{References}

1. Rey E, Lago-Ballesteros J, Padrón-Cabo A. Timing and tactical analysis of player substitutions in the UEFA Champions League. Int J Perform Anal Sport 2015; 15(3): 840-850. [doi: 10.1080/24748668.2015.11868835]
2. Harper LD, Fothergill M, West DJ, et al. Practitioners' perceptions of the soccer extra-time period: implications for future research PLoS One 2016; 11(7): e0157687. [doi:10.1371/journal.pone.0157687]

3. Harper LD, West DJ, Stevenson E, et al. Technical performance reduces during the extra-time period of professional soccer match-play. PloS One 2014; 9(10): e110995. [doi:10.1371/journal.pone.0110995]

4. Lago Peñas C, Dellal A, Owen AL, et al. The influence of the extra-time period on physical performance in elite soccer. Int J Perform Anal Sport 2015; 15(3): 830-839. [doi: 10.1080/24748668.2015.11868834]

5. Russell M, Sparkes W, Northeast J, et al. Responses to a $120 \mathrm{~min}$ reserve team soccer match: a case study focusing on the demands of extra time. J Sports Sci 2015; 33(20): 2133-2139. [doi: 10.1080/02640414.2015.1064153]

6. Winter C, Pfeiffer M. Tactical metrics that discriminate winning, drawing and losing teams in UEFA Euro 2012®. J Sports Sci 2016; 34(6): 486-492. [doi: 10.1080/02640414.2015.1099714]

7. Dmitriy A, Mike V, Ilya V, et al. Validation and precision analysis of InStat Fitness system. InStat 2013; 1-14 http://www.instatscout.com

8. Carling C, Williams A, Reilly T. The handbook of soccer match analysis: a systematic approach to improving performance. London: Routledge, 2005:95.

9. Bradley PS, Sheldon W, Wooster B, et al. High-intensity running in English FA Premier League soccer matches. J Sports Sci 2009; 27(2): 159-168. [doi: 10.1080/02640410802512775]

10. Rampinini E, Impellizzeri FM, Castagna C, et al. Technical performance during soccer matches of the Italian Serie A league: effect of fatigue and competitive level. J Sci Med Sport 2009; 12(1): 227-233. [doi: 10.1016/j.jsams.2007.10.002] 